\title{
MICROABRASIÓN DE ESMALTE DENTAL: UNA ALTER- NATIVA DE TRATAMIENTO PARA LA FLUOROSIS. REPORTE DE UN CASO
}

\author{
Mercelena Sánchez Molina. \\ Odontóloga, U. Autónoma de Manizales, \\ Residente de I año de la Especialización en Odontopediatría, U. Santo Tomás
}

Autor responsable de la correspondencia: Mercelena Sánchez $M$.

Correo electrónico: mercelena odontopediatria@yahoo.es

Presentación realizada en el Concurso de Posters en el XIV Congreso Internacional de Odontología Pediátrica,

en Santa Marta, junio 4 de 2005

\begin{abstract}
RESUMEN
El ENSAB III, Estudio Nacional de Salud Bucal de 1998 al 2000, reportó una alta prevalencia de fluorosis en la población colombiana. La fluorosis se presenta como una hipomineralización del esmalte; causa defectos de pigmentación y textura que afectan la estética del paciente. La microabrasión es una alternativa poco invasiva que mejora la estética en los pacientes que presentan fluorosis dental leve a moderada. El objetivo de este artículo es presentar un caso clínico de una paciente con fluorosis, en la que se usó la técnica de microabrasión. [Sánchez M. Microabrasión de esmalte dental: una alternativa de tratamiento para la fluorosis. Reporte de un caso. Ustasalud Odontología 2005; 4: 116 - 121]
\end{abstract}

Palabras clave: Fluorosis, Microabrasión, Esmalte dental.

\section{MICROABRASION OF DENTAL ENAMEL: AN ALTERNATIVE TREATMENT FOR FLUOROSIS. REPORT OF A CASE}

\begin{abstract}
The National Study of Oral Health from 1998 to 2000, reported a high prevalence of fluorosis in the Colombian population. The fluorosis appears as a hipomineralization of the enamel; cause defects of pigmentation and texture that affect the aesthetic. Microabrasion is a little invasive alternative that improves the aesthetic appearance in the patients who present slight to moderate dental fluorosis. The objective of this article is to present a clinical case of a patient with fluorosis, in which the use of microabrasion improves her appearance.
\end{abstract}

Key words: Fluorosis, Microabrasion, Tooth enamel.

Recibido para publicación: 26 de mayo de 2005. Aceptado para publicación: 12 de noviembre de 2005.

\section{INTRODUCCIÓN}

La cantidad óptima de fluoruro se define en el equilibrio existente entre la reducción máxima en la prevalencia de caries y la ausencia de alteraciones en la formación del esmalte que causen problemas estéticos como la fluorosis dental. ${ }^{1}$

Estas alteraciones, usualmente, son bilaterales y simétricas. Comúnmente se ven afectados los incisivos superiores e inferiores, premolares y primeros molares permanentes. ${ }^{2-3}$
Hay pocos estudios sobre fluorosis en dientes deciduos aunque se argumenta que los dientes más afectados son los segundos molares primarios. Se ha determinado una etapa crítica de ingesta de flúor entre los 6 y 9 meses postnatales. $^{4 \cdot 5}$

Actualmente en Colombia, el índice de fluorosis se ha incrementado, relacionándose directamente con la alta ingesta de fluoruros en etapas del desarrollo y maduración de los dientes, aproximadamente desde el nacimiento hasta los ocho años de edad; en un rango de 0.03 a 0.1 $\mathrm{mg}$ de $\mathrm{F} / \mathrm{Kg}$. de peso por día. La manifestación de fluorosis 
ocurre en la etapa de maduración temprana del desarrollo del diente y continua hasta terminar su maduración. $^{6-7}$

En nuestro país, la fluorización en el agua se llevó a cabo hasta 1986, seguida por fluorización en la sal. En 1991 se llevó a cabo un estudio que reportó que, solamente, el $10 \%$ de la sal comercial, cumple con las exigencias óptimas de concentración de flúor (180 a 220ppm F/K de sal). ${ }^{8}$ Esta situación se suma a la ingesta de suplementos con flúor, alimentos, bebidas, cremas y enjuagues dentales fluorados, sin supervisión. ${ }^{6-8}$ Esto lleva a pensar en la fluorosis dental como un síntoma de intoxicación crónica. ${ }^{9}$

En el ENSAB III, 1998 al 2000, se determinó que la proporción de las personas con fluorosis, para las edades de 6, 7, 12 , y 15 a 19 años era de 11.5\%. La mayoría de las personas afectadas presentan fluorosis muy leve y leve; solamente, el $0.8 \%$ presentaban lesiones moderadas y severas. ${ }^{2}$

En cuanto a la prevalencia de esta patología, existe una diferencia importante, niños con edades entre los 6 y 7 años se vieron afectados en un $25.7 \%$, el segundo grupo conformado por niños de 12 años, fue afectado en un $18.7 \%$, y en los adolescentes de 15 a 19 años, se observó una disminución, 5.3\%. Estos datos sugieren una exposición diferente a los fluoruros para cada grupo de edad. Por otra parte, también se encontraron diferencias importantes entre las regiones de Colombia, siendo la región Central y la Atlántica las de mayor prevalencia. ${ }^{2}$

Es importante anotar, que también hubo diferencias notables, según el tipo de diente. Se determinó una mayor prevalencia en los premolares, seguido de los incisivos centrales, laterales y por último los caninos. ${ }^{2}$

Como soporte para un diagnóstico clínico, se han desarrollado dos índices que determinan el grado de fluorosis:

El índice de Dean, 1942, que se basa en seis diagnósticos: 0 . Normal.

1. Cuestionable.

2. Muy leve (áreas pequeñas, dispersas, irregulares en un $25 \%$ de la superficie del diente).

3. Leve (áreas más extensas en un $50 \%$ de la superficie del diente).

4. Moderada (toda la superficie del diente, superficie con atrición, a veces con manchas cafés).

5. Severa (Toda la superficie hipoplásica, manchas cafés dispersas, apariencia corroída)., ${ }^{2,3}$
En 1978, Thylstrup y Fejerskov, desarrollaron una propuesta diagnóstica basada en diagramas y expresada de la siguiente manera:

0 . Normal.

1. Líneas opacas blancas atravesando la superficie del diente.

2. Líneas opacas blancas más pronunciadas y frecuentemente unidas.

3. Unión de líneas blancas y áreas nubosas esparcidas sobre muchas partes de la superficie.

4. Marcada opacidad con apariencia de tiza blanca, parte de superficie expuesta a la atrición.

5. Superficie completa opaca, con pits de menos de 2 mm. de diámetro.

6. Pits pequeños fusionándose en esmalte opaco formando bandas de menos de $2 \mathrm{~mm}$. de altura vertical.

7. Esmalte con áreas irregulares de menos de la mitad de la superficie, remanente de tejido opaco intacto.

8. Pérdida de más de la mitad de la superficie del esmalte. 9. Pérdida mayor de la superficie del esmalte, con cambios en la forma anatómica, a veces con halo de esmalte opaco en cervical. ${ }^{3}$

Una de las dificultades más comunes a la que se enfrentan los profesionales de la salud bucal es el diagnóstico diferencial de las lesiones de mancha blanca y de la fluorosis dental leve. La diferencia radica en que, por lo general, las lesiones de mancha blanca se ubican en el margen gingival y tienen una opacidad más pronunciada que las líneas opacas de la fluorosis, que se pueden observar en la clasificación TF1 y $2 .^{3}$

Como alternativa para mejorar la estética de los pacientes con fluorosis o lesiones de mancha blanca, se ha descrito un procedimiento operatorio llamado microabrasión del esmalte. Esta técnica es extremadamente conservadora, y se realiza mediante la utilización de ácidos y/o abrasivos. ${ }^{10}$

El primero en describir esta técnica fue el Dr. Walter Kane en 1916. El utilizó ácido clorhídrico líquido al 18\% y calor para eliminar manchas cafés. Posteriormente, McCloskey modificó esta técnica y usó ácido clorhídrico al 18\% sin calor. Croll y Cavanaugh usaron el mismo ácido con igual concentración en combinación con piedra pómez. ${ }^{11}$

En 1993, Tong y colaboradores verificaron por microscopía de luz polarizada que una aplicación de ácido clorhídrico al $18 \%$ por 100 segundos, promueve un desgaste de 
$100+/-47$ micras y que asociado con piedra pómez, en el mismo periodo de tiempo, el desgaste podía llegar a ser hasta de $360+/-130$ micras. Por otra parte, al utilizar el ácido fosfórico al 37\% y el peróxido de hidrógeno, se observó un desgaste similar con variaciones del orden de $5.5+/-1.5$ micras, lo que lo hace una alternativa más segura y con una eficacia comparable..$^{10,12,15}$

En 1993, Croll sugiere que para evitar que el esmalte sea propenso a la desmineralización y colonización de Estreptococo mutans se debe aplicar flúor, pues un esmalte remineralizado, se torna más rígido después de la realización de este procedimiento. ${ }^{11}$

Para reducir los problemas de toxicidad del ácido clorhídrico y proporcionar más seguridad al paciente y al odontólogo, en 1989, la casa Premier, introduce el Prema ${ }^{\circledR}$, material con baja concentración de ácido y abrasivo de alta dureza y partículas pequeñas, suspendidas en un gel. ${ }^{11}$

Mondelli y colaboradores, en 1995, introducen la técnica de microabrasión con ácido fosfórico al 37\% y piedra pómez, aunque realmente, los pioneros fueron Powel y Craig en $1982 .{ }^{11}$

En el 2004, la casa comercial Ultradent, introduce para tratamientos de microabrasión, el Opalustre ${ }^{\circledR}$, cuya composición es ácido clorhídrico al 6.6\% y $900 \mathrm{mg} / \mathrm{Kg}$. de carbonato de sílice. Sus especificaciones recomiendan dispensar con su jeringa predocificada, $1 \mathrm{mml}$ de espesor del material en la superficie vestibular y realizar la abrasión con "Opal Cups», en un contrangulo de baja velocidad, por 60 segundos .Es recomendado en defectos menores de $0.2 \mathrm{~mm} .^{13}$

Según estudio realizado por Matos y colaboradores, se confirmó mediante microscopía de barrido, que el uso de instrumentos rotatorios de baja velocidad, ofrece, clínica-mente, mejores resultados estéticos en comparación con la aplicación manual, usando ácido fosfórico al 37\% y piedra pómez. ${ }^{10}$

Por otra parte, una investigación realizada por Dalzell y colaboradores en 1995, determinó que el incremento de pérdida del esmalte es directamente proporcional, al tiempo, al número de aplicaciones, a la presión, y a la combinación de estas variables. ${ }^{12}$

El espesor de los incisivos centrales, por vestibular, en el tercio incisal es de $1.12 \mathrm{~mm}$, en el tercio medio, $0.93 \mathrm{~mm}$ y en el tercio gingival, $0.49 \mathrm{~mm}$, valores que son similares para los incisivos laterales, pero que varían con respecto a los incisivos inferiores, pues están en los rangos de 1.02 $\mathrm{mm}, 0.87 \mathrm{~mm}$, y 0.36 , respectivamente. Por lo tanto, se sugiere que un $25 \%$ a $30 \%$ de la reducción del esmalte puede ser, clínicamente, aceptable. ${ }^{12}$

Para obtener mejores resultados en la técnica de microabrasión, con Prema ${ }^{\circledR}$, Dalzer y colaboradores recomiendan una presión ligera (10 a $30 \mathrm{~g}$ ) que se logra con la presión del dedo en el instrumento, una menor cantidad de aplicaciones del compuesto microabrasivo y una menor exposición del diente tratado a tales compuestos, determinándose el tiempo máximo de cada aplicación en 20 segundos y de 6 a 8 aplicaciones a lo largo del tratamiento. ${ }^{11,12}$

Para la realización de esta técnica es determinante la etiología, debido a que el éxito del tratamiento depende de la profundidad de la lesión. Los defectos del color no siempre dan información adicional de la causa de la anomalía ya que la diferencia del color puede ser por poros en el esmalte que toman los pigmentos después de la erupción. ${ }^{14}$

\section{REPORTE DE CASO}

Paciente de sexo femenino, 8 años de edad, procedente de Palmira (Valle), ciudad ubicada en la región occidental de Colombia. Acude a las clínicas odontológicas de la Universidad Santo Tomás de Floridablanca por presentar manchas blancas en sus dientes permanentes.

No reporta ningún antecedente sistémico. Su madre reporta ingesta de crema dental con alto contenido en flúor y sal fluorada en su infancia temprana. Al examen clínico se observan manchas blancas generalizadas en incisivos centrales superiores, en laterales en erupción, en los cuatro incisivos inferiores y en los cuatro primeros molares permanentes, compatibles con un estado de fluorosis (Figura 1, 2 y 3).

Al realizar los índices determinantes de fluorosis dental se llegó al siguiente diagnóstico:

- Incisivos centrales superiores: Según el índice de Dean se clasifica como una Fluorosis Moderada y de acuerdo con el índice TF, un grado 5 .

- En los incisivos laterales, no se determinó porque aún están en erupción. 


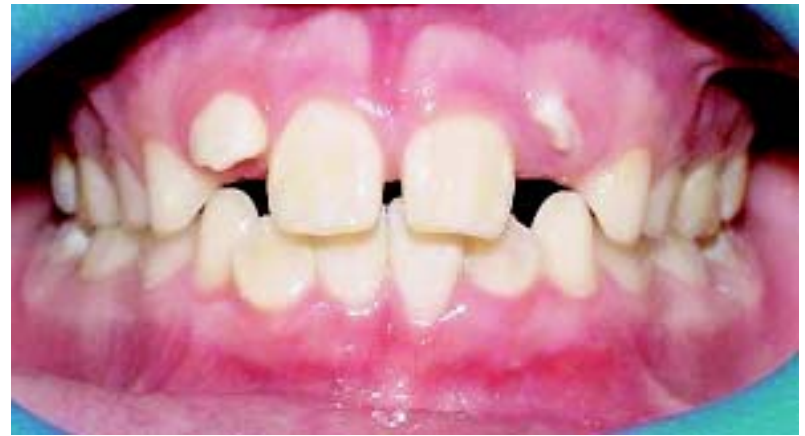

Figura 1. Examen intraoral.

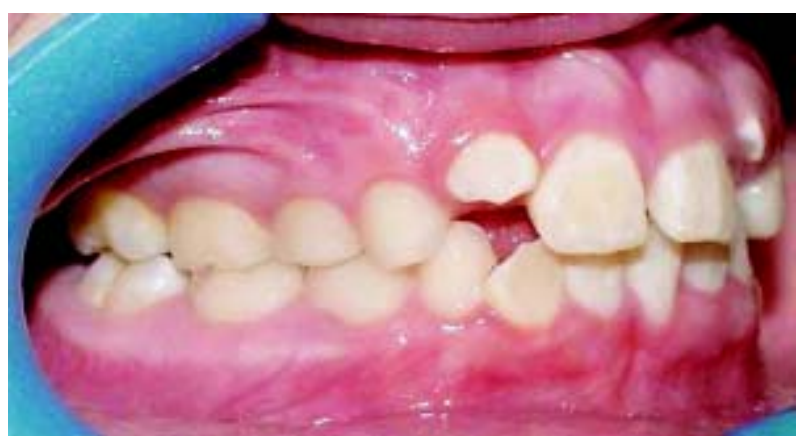

Figura 2. Oclusión lateral derecha.



Figura 3. Oclusión lateral izquierda.

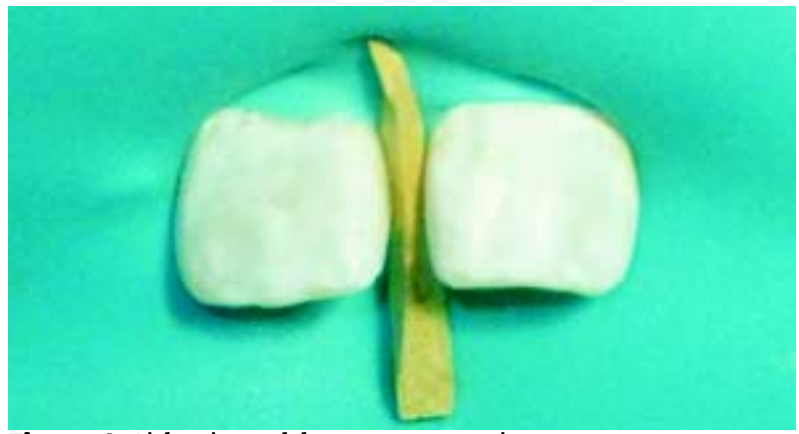

Figura 4. Aislamiento del campo operatorio.



Figura 5. Ácido fosfórico al 37\% y piedra pómez.

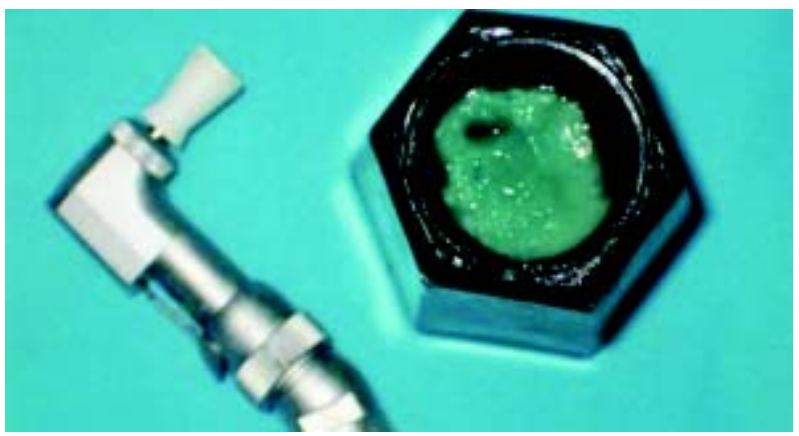

Figura 6. Preparación de la mezcla.

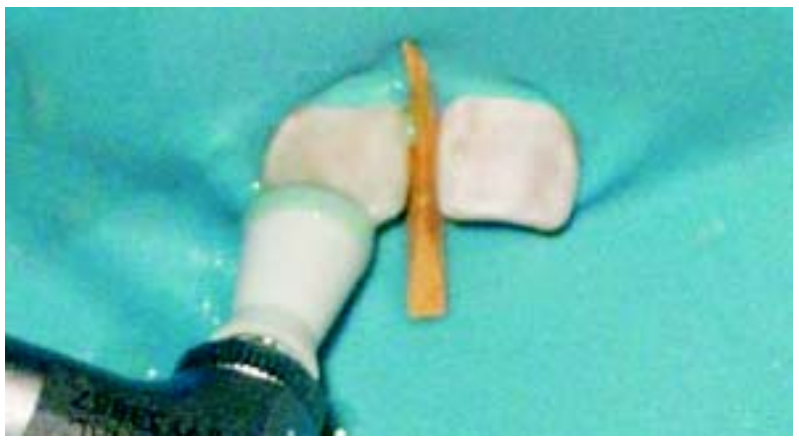

Figura 7. Aplicación de la mezcla.

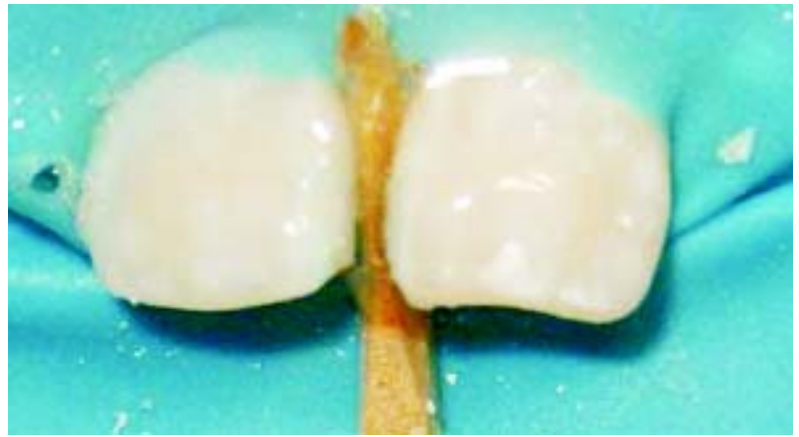

Figura 8. Aplicación de flúor neutro. 


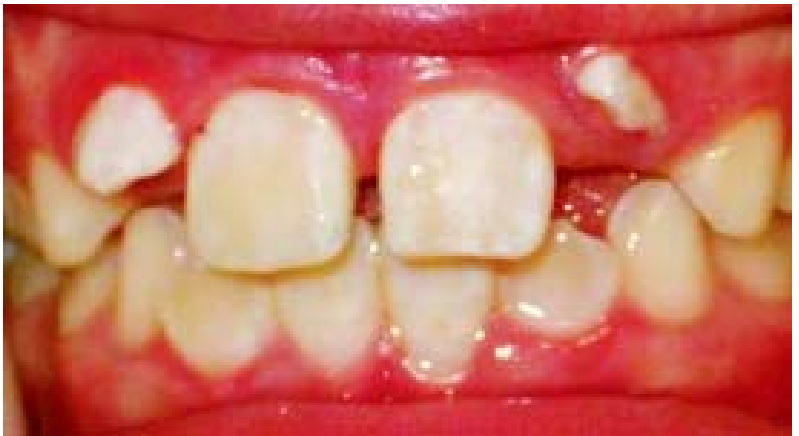

Figura 9. Antes del procedimiento.



Figura 10. Después del procedimiento.

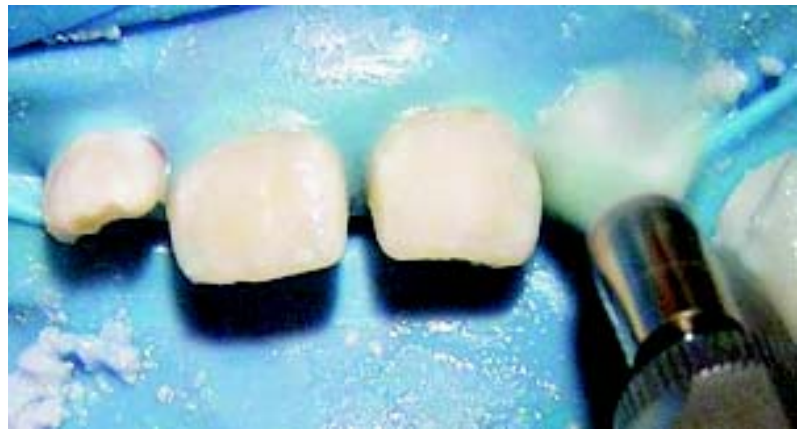

Figura 11. Sesión a los cuatro meses (abril de 2005).



Figura 12. Finalización de la sesión (abril de 2005).
- Primeros molares permanentes: Según Dean se clasifica como una Fluorosis Moderada y de acuerdo al índice TF, un grado 4.

- Incisivos inferiores:Según el índice de Dean se clasifica como una Fluorosis Leve y de acuerdo al índice TF, un grado 3.

Se decide realizar como tratamiento estético, microabrasión con ácido fosfórico y piedra pómez:

1. Se realizó aislamiento absoluto con tela de caucho en el 11 y en el 21 (Figura 4).

2. Se preparó una mezcla de ácido fosfórico al $37 \%$ y piedra pómez, en relación 1:1, hasta adquirir una consistencia cremosa (Figura 5, 6).

3. Se realizó la aplicación de la mezcla en tres intervalos de 15 segundos cada uno, con copa de caucho a baja velocidad (Figura 7).

4. Se llevó a cabo lavado profuso con agua.

5. Se secó con jeringa triple.

6. Por último se aplicó flúor neutro por 4 minutos (Figura 8). Al día siguiente se realizó el mismo procedimiento, con igual seguimiento (Figura 9 y 10).

La paciente regresa a los cuatro meses y se realiza una nueva sesión, especialmente sobre los laterales ya erupcionados (Figura 11 y 12).

\section{DISCUSIÓN}

Durante la etapa de maduración del desarrollo del diente, el agua y las proteínas son removidas de la matriz extracelular del esmalte, lo que permite el incremento de cristales de hidroxiapatita. Los efectos de la hipomineralización están asociados con la retención del $2 \%$ al $5 \%$ de proteínas de la matriz del esmalte, mientras que en un esmalte normal, la retención es, solamente, del $0.01 \%$ al $1 \%{ }^{11}$

La hipomineralización, puede ser la consecuencia de muchos desórdenes caracterizados por alteraciones en la estructura del esmalte. Estas condiciones incluyen defectos hereditarios, como la amelogénesis imperfecta 0 alteraciones sistémicas como en el caso de la fluorosis. ${ }^{11}$

La etiología de estas lesiones se basa en la historia dental, distribución de los dientes afectados y en la historia familiar. ${ }^{11}$

En este caso, la hipomineralización se presentó con una distribución similar en sus dientes homólogos y en dientes cuyo tiempo de maduración se encuentra en el mis- 
mo periodo (incisivos y primeros molares); no hay historia de trauma, ni antecedentes familiares con padecimiento de esta patología. Además, no hay enfermedades sistémicas, que puedan causar hipoplasia en el esmalte. Por lo tanto, este análisis llevó al diagnóstico de una fluorosis, que no sería de extrañar, debido a la alta prevalencia de esta patología en la población colombia$\mathrm{na},{ }^{2}$ por asociaciones con altas concentraciones de flúor en la sal de uso doméstico, pastas y enjuagues dentales fluorados usados por la paciente desde edades tempranas. ${ }^{8}$

Teniendo en cuenta el tratamiento propuesto, se podría sugerir que no está completamente claro el mecanismo exacto por el cual la microabrasión elimina los problemas de pigmentación del esmalte y mejora su apariencia. Sin embargo, puede ser que durante la microabrasión, el ácido disuelva el material orgánico residual, incluida la pigmentación y, posteriormente, el flúor y los minerales salivales, intensifiquen su remineralización. ${ }^{10,11}$

Otra posible explicación para el éxito del tratamiento, es el hecho de que la superficie microabrasionada, refleja y refracta la luz en los dientes, de tal forma que las imperfecciones leves del esmalte son borradas. ${ }^{11}$

En la paciente, hubo una reducción de las manchas, pero no en su totalidad por tratarse de una lesión moderada, según Dean, y una lesión grado 5, según el índice TF, su profundidad pigmentaria era bastante marcada. ${ }^{3}$

En cuanto a la textura, el cambio fue radical, pues hubo eliminación completa de los pits, y quedó una superficie totalmente lisa con el uso de la copa de caucho, como demostró el estudio basado en microscopía electrónica, de Matos, en $1998 .{ }^{10}$

\section{CONCLUSIONES}

1. La microabrasión es una técnica conservadora, que proporciona una mejor estética al paciente, sobre todo, en lesiones pigmentarias y morfológicas poco profundas, como en alteraciones leves de hipomineralización.

2. Debido a la alta prevalencia de fluorosis en nuestra población, se puede pensar en este tipo de tratamiento, antes de utilizar una técnica invasiva como coronas o carillas estéticas.

\section{BIBLIOGRAFÍA}

1. Thylstrup A, Fejerskow O. Efecto del fluoruro sobre la placa dental, la estructura del diente y la caries dental. En: Thylstrup A, Fejerskow O. Caries. Barcelona: Doyma; 1988.

2. República de Colombia. Ministerio de Salud. Centro Nacional de Consultoría. III Estudio Nacional de salud Bucal, ENSAB III, Dirección Nacional de Promoción y Prevención, 1999.

3. Fejerskov 0, Manji F, Baelum V. Indices for measuring dental fluorosis. En: Fejerskov 0, Manji F, Baelum V. Dental fluorosis - a handbook for health Workers - Munksgaard, Copenhagen: Munksgaard; 1988.

4. Leverett D.H.Madar S.M. Vaughan B.W. Proskin H.M. Moss M.E Randomized clinical trial of the effect of prenatal fluoride supplements in preventing dental caries. Caries Res 1997; 31: 174-179.

5. Levy SM, Hillis SL, Warren JJ, Broffitt BA, Mahbubul Islam AKM, Wefel $\mathrm{J}$, Kanellis MJ. Primary tooth fluorosis and fluoride intake during the first year of life. Community Dent Oral Epidemiol 2002; 30: 286 - 295.

6. Billings RJ, Berkowitz RJ, Watson G. Teeth. Pediatrics 2004; 113: 1120 - 1127.

7. Moisés SJ, Moisés ST, Allegretti ACV, Argenta M, Werneck R. Fluorose dental: ficcâo epidemiológica? Pan Am J Public Health 2002; 12: 339 - 346.

8. Galindo F. La fluorosis dental, nueva enfermedad de la niñez colombiana. Revista de la Academia Colombiana de Odontología Pediátrica 1998; $2: 5$ - 7.

9. Gonzáles MC. Efectos sistémicos del flúor en Colombia. Revista de la Academia Colombiana de Odontología Pediátrica 1998; 2: 10 - 14.

10. Matos A, Turbino M, Lacalle ME. Efeito das técnicas de microabrasao no esmalte: estudo em microscopia eletronicas de varredura. Rev Odontol Univ Sao Paulo 1998; 12: 105 -111.

11. Ashkenazi M. Sarnat H. Microabrasion of teeth with discoloration resembling hypomaduration enamel defects: four- year follow up. J Clin Pediatr Dent 2000; 25: 29 -33.

12. Dalzell DP, Howes RI, Hubler PM. Microabrasion: effect of time, number of applications, and pressure on enamel loss. Pediatr Dent 1995; 17: 207 - 211

13. Ultradent products inc. Opalustre. Enamel microabrasion slurry. [En línea] 2001. URL disponible en: http://www.ultradent.com/ products/instructions/55424_opalustre.pdf.

14. Wong FSL, Winter GB. Effectiveness of microabrasion technique for improvement of dental aesthetics. Br Dent J 2002; 193: 155 -158.

15. Tong LS, Pang MK, The effects of etching, micro-abrasion, and bleaching on surface enamel. J. Dent Res. 1993 Jan; 72 (1): 67-71. 